\title{
Assessing the outcome and influencing factors of a behavioral tobacco cessation intervention within a workplace setting: A mixed methods study
}

\author{
Gauri Mandal ${ }^{1}$, Srinath Satyanarayana ${ }^{2}$, Amol R. Dongre ${ }^{3}$, Thulasingam Mahalakshmy ${ }^{4}$, Himanshu A. Gupte ${ }^{5}$
}

\begin{abstract}
AFFILIATION
1 Salaam Bombay Foundation, Mumbai, India

2 The Union South-East Asia Office, New Delhi, India

3 Department of Extension Programme (SPARSH), Community Medicine and Medical Education, Pramukhswami Medical College (PSMC), Karamsad, India 4. Department of Preventive and Social Medicine, Jawaharlal Institute of Postgraduate Medical Education and Research, Puducherry, India

5 Narotam Sekhsaria Foundation, Mumbai, India
\end{abstract}

CORRESPONDENCE TO

Gauri Mandal. Salaam Bombay Foundation, 1st Floor Nirmal Building,

Popul. Med. 2021;3(June):16
Nariman Point, Mumbai, 400021, India. E-mail: gauri.mandal34@gmail. com ORCID ID: https://orcid.org/0000-0001-5533-9851

\section{KEYWORDS}

workplace, mixed methods, smokeless tobacco, tobacco use cessation, behavioral intervention

Received: 28 September 2020, Revised: 5 April 2021, Accepted: 5 April 2021

https://doi.org/10.18332/popmed/135443

\begin{abstract}
INTRODUCTION India has 267 million adult tobacco users, the majority of whom are part of the economically productive workforce. Workplaces are ideal settings to influence tobacco use behavior. A 6-month tobacco cessation program was implemented in five workplaces in Maharashtra, India. The study aimed to describe 6-month tobacco cessation outcomes and to identify promoters and barriers for implementing the program.

METHODS This is an explanatory mixed methods study. Quantitative part involved analysis of routinely collected program data from January 2016 to June 2017 to describe the sociodemographic characteristics, tobacco use patterns and 6-month cessation outcomes. Qualitative part consisted of one-to-one interviews with employees who had enrolled for cessation services $(n=16)$ and one group interview with the counseling service providers $(n=5)$.
\end{abstract}

\section{INTRODUCTION}

The epidemic of tobacco use is one of the greatest threats to global health today. Tobacco use is one of the main risk factors for a number of chronic diseases such as cancers, lung diseases, and cardiovascular diseases ${ }^{1}$. Worldwide, it is estimated that about six million people die prematurely every year, and if the current trends continue, about one billion people will die prematurely during this century as a result of tobacco use. Of these deaths, $80 \%$ will occur in lowand middle-income countries ${ }^{1}$.

India is the second largest consumer of tobacco ${ }^{2}$. According to Global Adult Tobacco Survey-2 (GATS-2), 267
RESULTS A total of 331 current tobacco users voluntarily enrolled in this program and used predominantly (78\%) smokeless tobacco. The self-reported 7-day point prevalence abstinence at the end of six months was $66 \%$. Employees who used smokeless forms of tobacco, had low levels of nicotine dependence, and attended 4 or more counseling sessions, were more likely to quit tobacco use. Support from the management and multiple counseling sessions were described as promoters. Peer pressure, work stress, and inability to quit without medication were the major barriers. Formulating tobacco-free workplace policy was suggested for reducing tobacco use at workplaces.

CONCLUSIONS The core activities of the program, which included awareness sessions, individual counseling sessions and repeated follow-up sessions as well as support received from the workplace management, played a major role in achieving good cessation outcomes.

million adults (who form a major part of the workforce) in the country consumed tobacco in some form ${ }^{3,4}$. Here, tobacco is consumed in two forms (smoking and smokeless) with the prevalence of smokeless tobacco (21.4\%) double that of the smoking $(10.7 \%)^{3,5}$.

The International Classification of Diseases (ICD-10) recognizes 'tobacco dependence' as a disease ${ }^{6}$. Global evidence shows that about $40 \%$ of current smokers attempt to quit each year on their own, and of these only about $4 \%$ to $6 \%$ are successful ${ }^{7}$. In India, according to GATS-2, 55\% of smokers and $50 \%$ of smokeless tobacco users wanted to quit tobacco-use ${ }^{3}$. Therefore, providing tobacco cessation services 
in India is recommended as a priority health intervention ${ }^{8}$.

Recent evidence provides stronger support for counseling (both when used alone and with other medical therapies) as an effective tobacco cessation strategy ${ }^{9}$. However, very little is known about the effectiveness of these interventions in people who use smokeless tobacco, the most prevalent form of tobacco use in India ${ }^{10,11}$.

Workplaces are ideal to reach a large number of people and influence their tobacco use behavior ${ }^{12}$. Workers spend most of their interactive hours in a day at the workplace ${ }^{13}$. A previous study from Maharashtra showed that tobacco cessation services provided at workplaces achieved better outcomes than clinic-based cessation services ${ }^{14}$. Therefore, in India, it is crucial to design and implement workplace tobacco cessation interventions.

LifeFirst, a collaborative initiative of two NonGovernmental Organizations - Narotam Sekhsaria Foundation (NSF) and Salaam Bombay Foundation (SBF) - provides tobacco cessation services at workplaces. We undertook an operational research study which was conducted under programmatic conditions in five different workplaces in Maharashtra. The study had two objectives: First, to describe the sociodemographic characteristics, tobacco use pattern, level of adherence to the cessation protocol and tobacco cessation rates of employees who enrolled in the LifeFirst cessation program from January 2016 to June 2017. Second, to understand the facilitators and challenges faced by the employees for quitting tobacco use and by the counsellors in providing cessation services at workplaces.

\section{METHODS}

\section{Study Design}

We used an explanatory mixed method design where the quantitative data collection (a cohort study) was followed by qualitative data collection.

\section{Study setting}

General setting

The study was conducted in the state of Maharashtra. It is India's second-most populous state and also the most industrialized state of the country contributing $13 \%$ of the national industrial output ${ }^{15}$. Of all adult inhabitants in Maharashtra, 27\% are tobacco users, of whom $91 \%$ use smokeless tobacco $^{16}$.

\section{Specific setting}

LifeFirst cessation services were provided in five workplaces selected purposively. A few workplaces in Maharashtra were approached and those which agreed to implement this program were selected.

\section{LifeFirst protocol}

LifeFirst follows a standard protocol, based on the American Cancer Society's guideline ${ }^{17}$ and WHO's 5As and
5Rs package ${ }^{18}$ with minor local adaptations. At the outset, awareness sessions are conducted for all the employees to sensitize them about the harms of tobacco use and to encourage the tobacco users to enroll for cessation services. Subsequently, individual counseling sessions are conducted for all the enrolled current tobacco users. During the first session, a counsellor asks about patterns and frequency of tobacco use, assesses nicotine dependence using the Fagerström scale for smokers ${ }^{19}$ and smokeless tobacco users ${ }^{20}$, encourages the user to quit, charts out their treatment plan and suggests different coping techniques. With the help of behavior modification techniques and motivational interviewing, the counsellor assists them for a successful quit attempt. After the first counseling session, each employee attends five follow-up counseling sessions at 15 days, one month, two months, four months and six months from the date of enrolment. The program is provided within the working hours in the workplace. The LifeFirst protocol and its activities are given in Table 1.

The self-reported tobacco use status is recorded and 7-day Point Prevalence Abstinence (PPA) ${ }^{21,22}$ is calculated at each follow-up. The operational definitions related to the study populations and their cessation outcomes are given in Table 2.

\section{Study population}

The study population included employees who were current tobacco users and who voluntarily enrolled in the LifeFirst workplace tobacco cessation services from January 2016 to June 2017.

The qualitative data was collected from five counsellors and 16 employees who had enrolled in this program. These employees were purposefully selected based on whether they were using $(n=7)$ or not using $(n=9)$ tobacco at six months.

\section{Data variables, sources of data and data collection Quantitative part}

Data of all the employees who enrolled in the program from January 2016 to June 2017 were collected using a counseling intake sheet and follow-up sheets after obtaining written informed consent. This was entered into Microsoft Excel and maintained as a database.

\section{Qualitative part}

All interviews were conducted in a language which was understood by the participants by GM (female) with mentorship from AD. Both these authors are well qualified in qualitative research.

Interviews with employees enrolled in counseling services To understand 'facilitators' and 'challenges' in stopping tobacco use at the workplace from employees' point of view, phone one-to-one interviews were conducted using an interview guide (Supplementary file), after obtaining informed consent, until saturation was attained. The average 
Table 1. LifeFirst workplace tobacco cessation protocol and activities implemented in five workplaces of Maharashtra from January 2016 to June 2017

$\quad$ Awareness $\rightarrow$
Conducted in groups for all the
employees.
The following topics were
covered during the awareness
session:
- Types of tobacco: smoking
and smokeless
- Long- and short- term ill
effects of tobacco
- Health, financial and social
benefits of quitting
- Health effects of smokeless
tobacco
- Symptoms of pre-cancerous
lesions and oral cancer
- LifeFirst program counseling
services

PowerPoint presentations or flip charts and videos in Hindi, English or local language were used as per the employee group.

Education material/leaflet containing tobacco related facts, health effects and benefits of quitting was also distributed.

\section{Registration $\rightarrow$}

The employees who were current tobacco users were encouraged to voluntarily register for the cessation services.

services.

.

(n) 
Table 2. Operational and cessation outcome definitions used during implementation of LifeFirst workplace tobacco cessation program in five workplaces of Maharashtra from January 2016 to June 2017

\begin{tabular}{|c|c|}
\hline Term & Operational definitions \\
\hline \multicolumn{2}{|l|}{ General } \\
\hline Employee & A person employed in a workplace for daily wage or salary \\
\hline Permanent employee & $\begin{array}{l}\text { Employee on the payroll of the workplace. They usually include supervisory staff involved } \\
\text { in planning and implementation }\end{array}$ \\
\hline Management employee & $\begin{array}{l}\text { Employee who is responsible for overall functioning of an organization and are the } \\
\text { decision makers }\end{array}$ \\
\hline Contractual employee & People taken up for specific tasks for a specified period \\
\hline Current user & Employee who has consumed any form and quantity of tobacco in the last 30 days \\
\hline 7-day Point Prevalence Abstinence & $\begin{array}{l}\text { Proportion of tobacco users who have not consumed tobacco in any form in the last seven } \\
\text { days }\end{array}$ \\
\hline Successful follow-up & If the counsellor was able to interact with the employee on the day of the visit \\
\hline Unsuccessful follow-up & If the employee was absent during the follow-up visit or has left the job \\
\hline \multicolumn{2}{|l|}{ Tobacco cessation outcome } \\
\hline Not using & Employee has not used any form of tobacco in the last 7 days \\
\hline Reduced & $\begin{array}{l}\text { Employee has reduced the quantity or frequency of tobacco use compared to the } 1 \text { st } \\
\text { session }\end{array}$ \\
\hline No change & $\begin{array}{l}\text { Employee has not made any change in the quantity or frequency of tobacco use compared } \\
\text { to the 1st session }\end{array}$ \\
\hline Relapse & Employee had made a quit attempt but restarted tobacco use \\
\hline Increased & $\begin{array}{l}\text { Employee has increased the quantity or frequency of tobacco use compared to the 1st } \\
\text { session }\end{array}$ \\
\hline Left the job & Employee has left the workplace \\
\hline Withdrawn from service & Employee has opted out of the tobacco cessation service \\
\hline Lost to follow-up & Employee was not available for the last two follow-up sessions \\
\hline Absent & Employee was not available for the last follow-up session \\
\hline
\end{tabular}

$259(78 \%)$ employees who used smokeless tobacco, 38 $(12 \%)$ who smoked and $34(10 \%)$ who used both forms of tobacco. Their sociodemographic details disaggregated by their type of tobacco use are given in Table 3.

All enrolled employees were males. The majority were in the aged 25-44 years, had contractual employment, and were married.

Among the employees who used smokeless tobacco, tobacco-lime mixture was the most commonly used product (71\%) followed by kharra (a local product which is a combination of tobacco, areca nut, lime, and catechu that is used predominantly in Eastern Maharashtra) $)^{24}(21 \%)$ and gutkha $(20 \%)$. Among the smokers, cigarette was the most commonly used product (65\%) followed by bidis (37\%). Several tobacco users reported using more than one smoking or smokeless tobacco product. The contractual employees used predominantly smokeless (82\%) form of tobacco when compared to permanent employees who predominantly smoked (71\%).

The tobacco use pattern is presented in Table 4.
Among the 331 enrolled tobacco users, 323 (98\%) were using tobacco daily. Most smokers (75\%) had low nicotine dependence ${ }^{19}$. In employees using smokeless forms of tobacco, nearly half (53\%) had medium nicotine dependence. Prior to enrolment, 142 (43\%) had made at least one attempt in the last 12 months to quit tobacco but were not successful. After enrolment, 263 (79\%) of the enrolled employees attended at least 4 sessions.

Of all the enrolled employees, 16 (5\%) left their job after enrollment. Of the remaining 315 employees, at six months, $208(66 \%)$ self-reported that they have not used tobacco in the last seven days, $41(12 \%)$ had reduced their tobacco use, $68(16 \%)$ were either lost to follow-up or their outcomes could not be assessed, and 11 (3\%) had relapsed.

The crude and adjusted relative risks for the associations between sociodemographic factors and cessation outcome (7-day PPA) are given in Table 5.

In adjusted analysis, adherence to counseling sessions was strongly associated with quitting. The other factors that were associated with quitting were age $<25$ years and low nicotine 
Table 3. Sociodemographic characteristics of current tobacco users who enrolled in workplace tobacco cessation services at five workplaces under LifeFirst program in Maharashtra, from January 2016 to June 2017 (N=331)

\begin{tabular}{|c|c|c|c|c|c|c|c|c|}
\hline \multirow[t]{2}{*}{ Characteristics } & \multicolumn{2}{|c|}{ Total } & \multicolumn{2}{|c|}{ Smokeless } & \multicolumn{2}{|c|}{ Smoking } & \multicolumn{2}{|c|}{ Both } \\
\hline & $\mathbf{n}$ & $\%$ & n & $\% *$ & $\mathbf{n}$ & $\% *$ & $\mathbf{n}$ & $\% *$ \\
\hline Total number of employees enrolled & 331 & 100 & 259 & 78 & 38 & 12 & 34 & 10 \\
\hline \multicolumn{9}{|l|}{ Age (years) } \\
\hline $15-24$ & 36 & 11 & 23 & 64 & 6 & 17 & 7 & 19 \\
\hline $25-34$ & 144 & 44 & 110 & 76 & 20 & 14 & 14 & 10 \\
\hline $35-44$ & 103 & 31 & 86 & 83 & 7 & 7 & 10 & 10 \\
\hline$\geq 45$ & 48 & 14 & 40 & 83 & 5 & 10 & 3 & 7 \\
\hline \multicolumn{9}{|l|}{ Employment type } \\
\hline Permanent & 21 & 6 & 6 & 29 & 15 & 71 & 0 & 0 \\
\hline Contractual & 310 & 94 & 253 & 82 & 23 & 7 & 34 & 11 \\
\hline \multicolumn{9}{|l|}{ Education level } \\
\hline No formal schooling & 37 & 11 & 26 & 70 & 2 & 5 & 9 & 24 \\
\hline Primary school completed & 66 & 20 & 56 & 85 & 7 & 11 & 3 & 5 \\
\hline Less than secondary school & 49 & 15 & 45 & 92 & 1 & 2 & 3 & 6 \\
\hline Secondary school completed & 73 & 22 & 63 & 86 & 5 & 7 & 5 & 7 \\
\hline Higher secondary school completed & 61 & 18 & 45 & 74 & 3 & 5 & 13 & 21 \\
\hline College completed & 27 & 8 & 21 & 78 & 6 & 22 & 0 & 0 \\
\hline Postgraduate degree completed & 18 & 5 & 3 & 17 & 14 & 78 & 1 & 6 \\
\hline \multicolumn{9}{|l|}{ Marital status } \\
\hline Single & 31 & 9 & 20 & 65 & 9 & 29 & 2 & 6 \\
\hline Married & 300 & 91 & 239 & 80 & 29 & 10 & 32 & 11 \\
\hline
\end{tabular}

* Row percentage.

dependence. Smokers were less likely to quit when compared to those using smokeless forms.

\section{Qualitative}

Employee interviews

The major facilitators within the workplace for tobacco cessation in employees were: support from the workplace 'management', such as having 'no-tobacco use rule' within the workplace, ensuring that tobacco products are not carried by the employees by having security checks at the entrances, reinforcement of messages to quit tobacco during safety meetings by the supervisor.

'We have received very good support from the company. The company has stopped the consumption of tobacco. All the packets are thrown away at the gate.'

'Our supervisors keep telling us about the ill effects of tobacco at safety meetings.'

Awareness sessions regarding harms of tobacco use and counseling sessions conducted by the LifeFirst program were considered as facilitators by the employees.

'Sir [LifeFirst counselor] had informed us about ill effects of tobacco and showed us a video and after seeing that I did not feel like smoking.'
The major workplace related barrier for tobacco cessation described by the employees were peers who consumed tobacco, considered as a barrier because they not only consume tobacco but also offer tobacco to others.

'I am trying my level best to quit. If someone offers me, then only I eat. I have stopped keeping it [smokeless tobacco product] in my pocket.'

'There were many people who used to eat tobacco and after seeing them I also felt like eating.'

Lack of provision of medicines was considered as a barrier by a few employees because they perceived that medicines would have helped them to quit.

'Without taking anything [medicine], the habit will not go away.'

Last barrier reported was working in night shifts as some employees consumed tobacco to remain awake during night shift.

'During night shift, I used to feel sleepy and therefore used to eat kharra [local smokeless product]. After eating that my sleep used to go away.'

A few suggestions provided by the employees for preventing tobacco use at workplaces by the employees were that the management should formulate tobacco-free 
Table 4. Tobacco use patterns and cessation outcome of current tobacco users who enrolled in workplace tobacco cessation services at five workplaces under LifeFirst program in Maharashtra, January 2016 to June $2017(\mathrm{~N}=331)$

\begin{tabular}{|c|c|c|}
\hline Characteristics & \multicolumn{2}{|c|}{ Values } \\
\hline \multirow[t]{2}{*}{ Age of initiation (years), mean \pm SD } & \multicolumn{2}{|c|}{$22.81 \pm 7.95$} \\
\hline & $\mathbf{n}$ & $\%$ \\
\hline \multicolumn{3}{|l|}{ Type of tobacco use } \\
\hline Smokeless & 259 & 78 \\
\hline Smoking & 38 & 12 \\
\hline Both & 34 & 10 \\
\hline \multicolumn{3}{|l|}{ Tobacco use } \\
\hline Daily & 323 & 98 \\
\hline Less than daily & 8 & 2 \\
\hline
\end{tabular}

Fagerström scale tobacco dependence

\section{Smoking}

Low

$54 \quad 75$

Medium

$14 \quad 19$

High

46

\section{Smokeless}

$\begin{array}{lrr}\text { Low } & 104 & 35 \\ \text { Medium } & 154 & 53 \\ \text { High } & 22 & 8 \\ \text { Missing } & 13 & 4\end{array}$

\section{Past quit attempts}

\begin{tabular}{lrr}
0 & 163 & 49 \\
1 & 90 & 27 \\
$2-3$ & 46 & 14 \\
$\geq 4$ & 6 & 2 \\
Unknown & 2 & 1 \\
Missing & 24 & 7 \\
Counselling sessions attended & & \\
1 & 13 & 4 \\
2 & 23 & 7 \\
3 & 32 & 10 \\
4 & 50 & 15 \\
5 & 86 & 26 \\
6 & 127 & 38 \\
\hline Outcome at end of 6 months & & \\
Not using & 208 & 63 \\
Reduced & 41 & 12 \\
Lost to follow-up & 35 & 11 \\
Absent & 17 & 5 \\
Left the job & 16 & 5 \\
Relapse & 11 & 3 \\
Increased & 2 & 0.6 \\
No change & 1 & 0.4
\end{tabular}

workplace policy, conduct security-checks at the entrance gate to stop people from using tobacco, provide information about ill effects of tobacco during the safety talks/meetings, provide support to those who are trying to stop tobacco, recognize or reward those who have quit completely and medicines or tablets should be provided to support them in quitting.

'Tobacco should be completely banned from the company.'

'There should be rules and people must be informed.'

'If we can get something which can help to quit tobacco, any tablet.'

\section{Counsellor interview}

Initial support and facilitation from the management in organizing the awareness program and encouragement given to the employees for joining the cessation services was considered as a facilitator for implementing the program by the counselors.

'They supported us by giving a dedicated space for counseling, gave us phone, and sent reminder emails. They took the initiative of conducting floor walks, gathering the employees, making them available. This system where the HR took initiative really worked.'

Counselors also considered integrating tobacco cessation messages into the workplace's internal programs or activities as an opportunity and a facilitating factor.

'In a company, health camp is conducted every year. We integrated our activities with the health checkup. We could reach all the employees.'

The challenges faced by the counsellors were related to the process components of the program protocol. The first challenge reported by the counsellors was enrolment of all the tobacco users for the tobacco cessation program. This was due to misconceptions regarding the term counseling. Employees associated counseling with mental health in a negative sense.

'Whenever we used the term "counseling", this term only used to block the employees.'

'They feel that I don't have any psychiatric problem for which I require counseling.'

Hierarchy within the workplace was also one of the reasons.

'Management staff feels that I am a management employee how I can join a program where the lower staff has already joined the program ... what will my colleagues say about me.'

The social image of the employees within the workplace acted as one of the reasons for tobacco users not enrolling into the program.

'So, if someone comes to the counseling room then people start thinking what has happened.'

'None of the females enrolled because of social stigma. There were few females who smoked but did not enroll in the program.'

The second challenge reported by the counsellors was that insufficient time was given to the employees by their supervisors for attending the counseling sessions. Unavailability of employees for the follow-up sessions was considered a challenge. Most of the contractual employees 
Table 5. Factors associated with the 7-day PPA at six months among remaining employees from those enrolled in workplace tobacco cessation services at five workplaces under LifeFirst program in Maharashtra from January 2016 to June 2017 ( $\mathrm{N}=315)$

\begin{tabular}{|c|c|c|c|c|c|c|c|c|}
\hline & Total & $\mathbf{n}$ & $\%$ & Crude RR & $95 \% \mathrm{CI}$ & Adjusted RR & $95 \%$ CI & $\mathbf{p}$ \\
\hline Total & 315 & 208 & 66 & & & & & \\
\hline \multicolumn{9}{|l|}{ Age (years) } \\
\hline $15-24$ & 33 & 24 & 73 & 1.09 & $0.90-1.32$ & 1.3 & 1.13-1.49 & $<0.001$ \\
\hline $25-34$ & 137 & 91 & 66 & Ref. & & Ref. & & \\
\hline $35-44$ & 97 & 56 & 58 & 0.87 & $0.72-1.04$ & 0.83 & $0.69-1.00$ & 0.053 \\
\hline$\geq 45$ & 48 & 37 & 77 & 1.16 & $0.95-1.41$ & 1.08 & $0.94-1.24$ & 0.266 \\
\hline \multicolumn{9}{|l|}{ Education level } \\
\hline No formal schooling & 34 & 25 & 74 & 1.04 & $0.77-1.40$ & 1.11 & $0.94-1.31$ & 0.197 \\
\hline Primary school completed & 65 & 41 & 63 & 0.89 & $0.73-1.08$ & 1.03 & $0.84-1.25$ & 0.764 \\
\hline Less than secondary school & 45 & 28 & 62 & 0.88 & $0.75-1.02$ & 0.94 & $0.83-1.08$ & 0.436 \\
\hline Secondary school completed & 68 & 48 & 71 & Ref. & & Ref. & & \\
\hline $\begin{array}{l}\text { Higher secondary school } \\
\text { completed }\end{array}$ & 59 & 33 & 56 & 0.79 & $0.63-0.98$ & 0.95 & $0.71-1.26$ & 0.728 \\
\hline College completed & 26 & 19 & 73 & 1.03 & $0.72-1.48$ & 1.18 & $0.99-1.41$ & 0.052 \\
\hline $\begin{array}{l}\text { Postgraduate degree } \\
\text { completed }\end{array}$ & 18 & 14 & 78 & 1.1 & $0.83-1.44$ & 1.19 & $0.91-1.55$ & 0.183 \\
\hline \multicolumn{9}{|l|}{ Type of employment } \\
\hline Permanent & 21 & 13 & 62 & Ref. & & Ref. & & \\
\hline Contractual & 294 & 195 & 66 & 1.07 & $0.89-1.28$ & 0.89 & $0.69-1.13$ & 0.344 \\
\hline \multicolumn{9}{|l|}{ Marital status } \\
\hline Single & 27 & 16 & 59 & 0.88 & $0.77-1.02$ & 0.88 & $0.74-1.04$ & 0.140 \\
\hline Married & 288 & 192 & 67 & Ref. & & Ref. & & \\
\hline \multicolumn{9}{|l|}{ Type of tobacco } \\
\hline Smokeless & 246 & 168 & 68 & Ref. & & & & \\
\hline Smoking & 38 & 23 & 61 & 0.88 & $0.72-1.09$ & 0.69 & $0.57-0.82$ & $<0.001$ \\
\hline Both & 31 & 17 & 55 & 0.8 & $0.52-1.24$ & 0.97 & $0.76-1.23$ & 0.825 \\
\hline \multicolumn{9}{|l|}{$\begin{array}{l}\text { Tobacco dependence at } \\
\text { baseline }\end{array}$} \\
\hline Low & 129 & 94 & 73 & 1.13 & $1.05-1.21$ & 1.2 & $1.10-1.30$ & $<0.001$ \\
\hline Medium & 148 & 95 & 64 & Ref. & & & & \\
\hline High & 25 & 11 & 44 & 0.68 & $0.36-1.29$ & 0.7 & $0.39-1.27$ & 0.252 \\
\hline Missing & 13 & 8 & 62 & 0.95 & $0.78-1.17$ & 1.15 & 0.99-1.35 & 0.066 \\
\hline \multicolumn{9}{|l|}{ Past quit attempts } \\
\hline 0 & 155 & 103 & 66 & Ref. & & & & \\
\hline 1 & 83 & 58 & 70 & 1.05 & $0.86-1.27$ & 0.94 & $0.80-1.12$ & 0.541 \\
\hline$\geq 2$ & 51 & 33 & 65 & 0.97 & $0.71-1.31$ & 0.98 & $0.80-1.20$ & 0.920 \\
\hline Unknown & 26 & 14 & 54 & 0.81 & $0.68-0.95$ & 0.84 & $0.66-1.07$ & 0.181 \\
\hline \multicolumn{9}{|l|}{$\begin{array}{l}\text { Counseling sessions } \\
\text { attended }\end{array}$} \\
\hline$<4$ & 57 & 16 & 28 & Ref. & & & & \\
\hline 4 & 45 & 28 & 62 & 2.21 & $1.43-3.43$ & 2.21 & 1.33-3.65 & 0.002 \\
\hline 5 & 86 & 60 & 70 & 2.48 & $1.67-3.68$ & 2.53 & $1.74-3.68$ & $<0.001$ \\
\hline 6 & 127 & 104 & 82 & 2.91 & $1.93-4.40$ & 3.00 & $2.09-4.30$ & $<0.001$ \\
\hline
\end{tabular}

PPA: point prevalence abstinence. RR: risk ratio. 
worked in production units or in places where the work could not be stopped, and therefore they could not leave their workstations. Some employees who wanted to attend the sessions were unable to do so because of their busy schedule. Some employees worked in night shifts and therefore their availability for follow-up sessions was difficult.

'They cannot switch off the machine, so we have to wait till their work is completed.'

'If the management staff is willing to come for the counseling session but is busy with meetings, trainings etc., it becomes impossible for them to attend the session. Work is important for them.'

Waning support from the management over a period of time was considered as a barrier in implementing the complete program.

'Initial reminders from the management supported but later on that support faded ... so if it could have been continuous ....'

The counselors mentioned that few action steps were taken by them to overcome few of these challenges during implementation. They conducted awareness and counseling sessions at the shop floor where the employees could not leave their workstations. A few of them conducted meetings and coordinated with the supervisors to change the shifts of the employees due for counseling sessions. Appointment system was used - mutually convenient time was fixed by the counsellor and employee for counseling sessions. They also reported that program activities were integrated with company activities like annual medical check-up, safety talks etc.

Advocacy and support for 'management' to formulate and implement tobacco-free workplace was suggested by the service providers as one of the solutions for addressing these barriers.

'If policy comes, the person who eats will also start thinking that I will have to quit.'

They also suggested that management should ensure continuous support to the employees for availing the cessation services and attending the follow-up counseling services on the designated date and time.

'The company and their management should provide time for the employees if the service providers come in the company.'

Lastly, they said that the cessation services should be repackaged to include more services such as 'stress management' and linkages for medical therapy to clients who are not responding to counseling.

'The program should give them a holistic treatment where along with cessation we provide stress management workshops, general workshop....'

\section{DISCUSSION}

During the program, 331 employees voluntarily enrolled in LifeFirst. High cessation rates were achieved with high adherence to follow-up counseling sessions. Support received from the management of workplace through repeated reminders from the supervisors, security checks, making employees available for counseling/awareness sessions were important facilitators and implementation of workplace tobacco-free policy was recommended.

The major strength of this study is the use of a mixed methods study design. For the quantitative part, we used data collected under routine programmatic settings without any exclusion. The analysis of previously collected programmatic data ensured that the study did not induce any improvements in the performance of the program. Therefore, the study findings reflect what has happened at the field level. The qualitative part of the study supplemented the quantitative findings and increased the understanding of several other practical issues. The study findings have the following implications.

First, the quantitative part of the study indicates that only males and predominantly contractual workers enrolled in the program. However, the qualitative part of the study revealed that there was a misconception with the term 'counseling' and misunderstanding among permanent employees, that the tobacco cessation services were predominantly for lower level/ contractual staff. The specific reason mentioned by a counselor for non-participation of female tobacco users was fear of disclosure of their tobacco use status. These findings imply that while implementing such interventions the abovementioned enrolment-related barriers should be addressed.

Second, there was a dose response relationship with those who attended more sessions achieving higher cessation outcomes. However, there were challenges in getting them to attend all the counseling sessions with less than half attending all the six counseling sessions. Support from the management and additional actions taken by the counselors to ensure that the employees were available for maximum follow-up sessions were crucial.

Third, the 6-month cessation outcomes achieved under this program were higher than those reported elsewhere $(6 \%$ to $40 \%)$ in other settings in India ${ }^{12,25,26}$. The major reasons for this are standard counseling protocol with one initial enrollment counseling followed by five individual face-to-face follow-up sessions over six months; ensuring compliance in attending the counseling sessions; support from management; and relatively lower levels of nicotine dependence in those who enrolled in our services. Association between low nicotine dependence at enrollment and high quit rate has been shown in previous studies ${ }^{27}$. Another Cochrane review concludes that providing intensive counseling support in the form of additional sessions improves cessation outcomes, which was also seen among employees who attended more sessions ${ }^{28}$.

Last reason for high cessation outcomes is higher proportion of smokeless tobacco users in our study population. A Cochrane systematic review shows that tobacco cessation services based on behavior change communication, such as counseling, help people quit smoking at workplaces ${ }^{29}$. However, there is no evidence of the effectiveness of the counseling services on smokeless 
forms of tobacco. Our study shows higher quit rates in smokeless users than in those who used smoked forms of tobacco. Therefore, this is new information and needs to be confirmed in larger studies.

In addition, previous studies also show education, age of starting tobacco use, gender, and frequency of tobacco use, to be associated with high quit rate $\mathrm{e}^{10,30}$.

Lastly, though the cessation outcomes were good and better than other studies, there was still scope for improvement in creating a facilitating environment for quitting tobacco use at the workplace. The study provides some suggestions from the employees' and the counsellors' perspectives on how to support employees in their quitting process further. Of these suggestions, we feel the suggestion pertaining to formulation of 'tobacco free' workplace policy to be the most pertinent and will achieve maximum effect on reducing the prevalence of tobacco use among employees.

The study also brings forth practical suggestions and action steps that are to be taken for smooth and successful implementation of a workplace tobacco cessation program. These can be incorporated into the existing guidelines ${ }^{17}$ (which currently focus on tobacco smoking and are suited more for developing countries) so that they can be implemented globally. Tobacco cessation for employees can be supported by government schemes or insurance policies which are currently non-existent.

\section{Limitations}

There are limitations in the study. First, the 6-month cessation outcomes were based on employee's self-report about their own tobacco use status. Biochemical validation was not possible due to budget restraints. Social desirability bias and pressure from the management may have played a role in this. Since there were no incentives or disincentives for wrong selfreporting, we feel the errors (if any) are likely to be minimal and random. Assessing the accuracy of self-reported outcome in this setting is an area for future research. Second, the qualitative interviews were done by the Investigator 1 who was a part of the LifeFirst tobacco cessation service. Therefore, the employees and other counsellors may not have given an honest/critical feedback about the deficiencies/inefficiencies of this program. Hence, the study may have missed some criticisms about the services. Third, 'support from the management' was identified as a critical factor for designing and implementing the tobacco cessation services at workplaces. However, we missed including persons from 'Management' in our qualitative research. As a result, we are unable to provide their perspectives about implementing workplace tobacco cessation services.

\section{CONCLUSIONS}

Good cessation outcomes were achieved by providing a counseling-based, on-site cessation service in workplaces. Multiple, face-to-face counseling sessions and support received from management played a major role. Tobacco cessation interventions are feasible in different workplace settings and should be promoted as a part of creating tobacco-free workplaces and integrated into other workplace initiatives.

\section{REFERENCES}

1. World Health Organization. WHO Report on the Global Tobacco Epidemic, 2008: The MPOWER package. 2008. Accessed April 5, 2021. https://apps.who.int/iris/bitstream/ handle/10665/43818/9789241596282_eng.pdf?sequence=1

2. Reddy KS, Gupta PC, eds. Report on Tobacco Control in India. Ministry of Health and Family Welfare, Government of India. November 25, 2004. Accessed April 5, 2021. https:// www.who.int/fctc/reporting/Annex6_Report_on_Tobacco_ Control_in_India_2004.pdf

3. Tata Institute of Social Sciences, Mumbai and Ministry of Health and Family Welfare, Government of India. Global Adult Tobacco Survey GATS 2 India 2016-17. 2018. Accessed April 5, 2021. https://ntcp.nhp.gov.in/assets/document/ surveys-reports-publications/Global-Adult-Tobacco-SurveySecond-Round-India-2016-2017.pdf

4. Census of India. Provisional Population Totals. Government of India; 2011. INDIA Series 1. Paper 1 of 2011. Accessed July 12, 2018. http://censusindia.gov.in/2011-prov-results/ data_files/india/paper_contentsetc.pdf

5. Bhan N, Karan A, Srivastava S, Selvaraj S, Subramanian SV, Millett C. Have Socioeconomic Inequalities in Tobacco Use in India Increased Over Time? Trends From the National Sample Surveys (2000-2012). Nicotine Tob Res. 2016;18(8):1711-1718. doi:10.1093/ntr/ntw092

6. World Health Organization. The ICD-10 Classification of Mental and Behavioural Disorders: Clinical Descriptions and Diagnostic Guidelines. World Health Organization; 1992. Accessed April 5, 2021. https://apps.who.int/iris/ bitstream/handle/10665/37958/9241544228_eng. pdf?sequence $=8$ \&isAllowed $=y$

7. Hughes JR. Motivating and helping smokers to stop smoking. J Gen Intern Med. 2003;18(12):1053-1057. doi:10.1111/j.1525-1497.2003.20640.x

8. Thankappan KR. Tobacco cessation in India: a priority health intervention. Indian J Med Res. 2014;139(4):484486. Accessed April 5, 2021. https://www.ijmr.org.in/temp/ IndianJMedRes1394484-4149243_113132.pdf

9. The Clinical Practice Guideline Treating Tobacco Use and Dependence 2008 Update Panel, Liaisons, and Staff. A clinical practice guideline for treating tobacco use and dependence: 2008 update. A U.S. Public Health Service report. Am J Prev Med. 2008;35(2):158-176. doi:10.1016/j.amepre.2008.04.009

10. Srivastava S, Malhotra S, Harries AD, Lal P, Arora M. Correlates of tobacco quit attempts and cessation in the adult population of India: secondary analysis of the Global Adult Tobacco Survey, 2009-10. BMC Public Health. 2013;13:263. doi:10.1186/1471-2458-13-263

11. Varghese C, Kaur J, Desai NG, et al. Initiating tobacco cessation services in India: challenges and opportunities. WHO South East Asia J Public Health. 2012;1(2):159-168. doi:10.4103/2224-3151.206929

12. Pimple S, Pednekar M, Mazumdar P, Goswami S, Shastri 
S. Predictors of Quitting Tobacco - Results of a Worksite Tobacco Cessation Service Program Among Factory Workers in Mumbai, India. Asian Pac J Cancer Prev. 2012;13(2):533538. doi:10.7314/apjcp.2012.13.2.533

13. Smedslund G, Fisher KJ, Boles SM, Lichtenstein E. The effectiveness of workplace smoking cessation programmes: a meta-analysis of recent studies. Tob Control. 2004;13(2):197-204. doi:10.1136/tc.2002.002915

14. Ransing RS, Patil DB, Desai MB, Modak A. Outcome of tobacco cessation in workplace and clinic settings: A comparative study. J Int Soc Prev Community Dent. 2016;6(5):487-492. doi:10.4103/2231-0762.192946

15. Economy of Maharashtra. Wikipedia. Updated May 25, 2021. Accessed July 10, 2018. https://en.wikipedia.org/wiki/ Economy_of_Maharashtra

16. World Health Organization. Global Adult Tobacco Survey (GATS): Fact Sheet, Maharashtraa 2016-17. Accessed June 13, 2018. https://tmc.gov.in/images/act/MaharashtraGATS2-Factsheet-Countryspecific-latest-v13.pdf

17. Global Smokefree Partnership. Smokefree-in-a-Box: A Guide for Companies Going Smokefree. 2008. Accessed April 5, 2021. https://www.cancer.org/content/dam/cancer-org/ smoke-free-worksites/smokefree-in-a-box-english.pdf

18. World Health Organization. Toolkit for delivering the 5A's and 5R's brief tobacco interventions in primary care. World Health Organization; 2014. Accessed April 5, 2021. https://apps.who. int/iris/bitstream/handle/10665/112835/9789241506953_ eng.pdf?sequence $=1$

19. Fagerstrom KO, Heatherton TF, Kozlowski LT. Nicotine addiction and its assessment. Ear Nose Throat J. 1990;69(11):763-765.

20. Directorate General of Health Services, Ministry of Health and Family Welfare, Government of India. Manual for Tobacco Cessation. Directorate General of Health Services, Ministry of Health and Family Welfare, Government of India; 2005. http://nimhans.ac.in/cam/sites/default/files/ Publications/Cancer_resource_Manual_4_Tobacco_New.pdf 21. Velicer WF, Prochaska JO. A comparison of four self-report

\section{ACKNOWLEDGEMENTS}

This research was conducted through the Structured Operational Research and Training Initiative (SORT IT), a global partnership led by the Special Program for Research and Training in Tropical Diseases at the World Health Organization (WHO/TDR). The model is based on a course developed jointly by the International Union Against Tuberculosis and Lung Disease (The Union) and Medécins sans Frontières (MSF/ Doctors Without Borders). The authors express their sincere thanks to the LifeFirst team and the management and employees of the workplaces for their participation in the study.

\section{CONFLICTS OF INTEREST}

The authors have each completed and submitted an ICMJE form for disclosure of potential conflicts of interest. The authors declare that they have no competing interests, financial or otherwise, related to the current work. G. Mandal reports that he is a full-time employee of Salaam Bombay Foundation and participation in the Structured Operational Research and Training Initiative (SORT IT). H. A. Gupte reports that he is a full-time employee of the Narotam Sekhsaria Foundation, and grants from the Clinton Global Initiative the Tobacco free workplace programme as a smoking cessation outcome measures. Addict Behav. 2004;29(1):51-60. doi:10.1016/s0306-4603(03)00084-4

22. Velicer WF, Prochaska JO, Rossi JS, Snow MG. Assessing outcome in smoking cessation studies. Psychol Bull. 1992;111(1):23-41. doi:10.1037/0033-2909.111.1.23

23. Tong A, Sainsbury P, Craig J. Consolidated criteria for reporting qualitative research (COREQ): a 32-item checklist for interviews and focus groups. Int J Qual Health Care. 2007;19(6):349-357. doi:10.1093/intqhc/mzm042

24. Bhisey RA. Chemistry and toxicology of smokeless tobacco. Indian J Cancer. 2012;49(4):364-372. doi:10.4103/0019-509X.107735

25. Savant SC, Hegde-Shetiya S, Agarwal D, Shirhatti R, Shetty D. Effectiveness of Individual and Group Counseling for Cessation of Tobacco Habit Amongst Industrial Workers in Pimpri, Pune - An Interventional Study. Asian Pac J Cancer Prev. 2013;14(2):1133-1139. doi:10.7314/apjcp.2013.14.2.1133

26. Mishra GA, Majmudar PV, Gupta SD, Rane PS, Uplap PA, Shastri SS. Workplace tobacco cessation program in India: A success story. Indian J Occup Environ Med. 2009;13(3):146153. doi:10.4103/0019-5278.58919

27. Lindberg A, Niska B, Stridsman C, Eklund BM, Eriksson $B$, Hedman L. Low nicotine dependence and high selfefficacy can predict smoking cessation independent of the presence of chronic obstructive pulmonary disease: a three year follow up of a population-based study. Tob Induc Dis. 2015;13(August):1-8. doi:10.1186/s12971-015-0055-6

28. Lancaster T, Stead LF. Individual behavioural counselling for smoking cessation. Cochrane Database Syst Rev. 2017;(3):CD001292. doi:10.1002/14651858.CD001292.pub3

29. Cahill K, Lancaster T. Workplace interventions for smoking cessation. Cochrane Database Syst Rev. 2014;(2):CD003440. doi:10.1002/14651858.CD003440.pub4

30. Gaikwad R, Bhowate R, Bajad P, et al. Potential Predictor of Tobacco Cessation among Factory Workers: A Baseline Data of Worksite Tobacco Cessation Programs in the Central Part of India. J Contemp Dent Pract. 2017;18(11):1071-1077. doi:10.5005/jp-journals-10024-2178

part of the Worksite Challenge.

FUNDING

The training program, within which this article was developed, was funded by the Department for International Development (DFID), UK, and La Fondation Veuve Emile Metz-Tesch, Luxembourg. The funders had no role in study design, data collection and analysis, decision to publish, or preparation of the manuscript. LifeFirst workplace tobacco cessation program implemented in the five workplaces was funded by Clinton Global Initiative Global Smokefree worksite challenge.

\section{ETHICAL APPROVAL AND INFORMED CONSENT}

Ethical approval for the study was obtained from the NSF and SBF Joint Ethics Committee, Mumbai, India and the Union Ethics Advisory Group (International Union against Tuberculosis and Lung Disease, Paris, France). All participants completed written informed consent.

\section{PROVENANCE AND PEER REVIEW}

Not commissioned; externally peer reviewed. 